\title{
VALIDAÇÃO DE INSTRUMENTO PARA REGISTRO DA SISTEMATIZAÇÃO DA ASSISTÊNCIA DE ENFERMAGEM PERIOPERATÓRIA
}

Validation of an instrument to register the systematization of perioperative nursing care

\author{
Validación de un instrumento para registrar la \\ sistematización de la atención de enfermería perioperatoria
}

Francielle Regina dos Santos Luciano ${ }^{*}$ (D), Luciana Martins da Rosa² (D), Ana Graziela Alvarez ${ }^{3}$ (D), Elisa Borges Kuze ${ }^{4}$ (D)

RESUMO: Objetivos: Construir e validar conteúdos de um instrumento para registro da sistematização da assistência de enfermagem perioperatória Método: Estudo metodológico realizado em um hospital-escola do sul do Brasil que incluiu revisão de literatura, mapeamento cruzado entre observação não estruturada e taxonomias da North American Nursing Diagnosis Association para construção do instrumento e aplicação da técnica Delphi para validação, realizada entre novembro e dezembro de 2018. Disponibilizou-se formulário eletrônico a dez expertos para avaliação da objetividade, clareza/compreensão, aparência e exequibilidade dos conteúdos do instrumento, registrada em escala Likert. As respostas obtidas foram submetidas ao índice de validade de conteúdo (IVC), e escores $\geq 0,8$ confirmaram a validação do conteúdo. Resultados: Os nove grupos de informações do instrumento foram avaliados por enfermeiros expertos. A média do IVC obtido entre todos os conteúdos foi de 0,92 na primeira rodada de validação. Os resultados demonstraram que a estratégia metodológica permitiu a construção de conteúdos que representam a necessidade clínica para os registros de enfermagem no período perioperatório. Conclusão: A implementação de instrumento validado contribui para uma prática de enfermagem mais segura e qualificada. Palavras-chave: Centros cirúrgicos. Cuidados de enfermagem. Lista de checagem. Processo de enfermagem. Segurança do paciente.

ABSTRACT: Objectives: To construct and validate the contents of an instrument to register the systematization of perioperative nursing care. Method: Methodological study conducted in a teaching hospital in Southern Brazil, which included literature review, cross-mapping between unstructured observation and North American Nursing Diagnosis Association taxonomies for instrument construction and application of the Delphi technique for validation, performed between November and December 2018. An electronic form was made available to ten experts to evaluate the objectivity, clarity/understanding, appearance and feasibility of the instrument contents, registered on a Likert scale. The answers obtained were submitted to the content validity index (CVI), and scores $\geq 0.8$ confirmed the content validation. Results: The nine information groups of the instrument were evaluated by expert nurses. The average CVI obtained among all contents was 0.92 in the first round of validation. The results showed that the methodological strategy allowed the construction of contents that represent the clinical need for perioperative nursing records. Conclusion: The implementation of a validated instrument contributes to a safer and more qualified nursing practice.

Keywords: Surgical centers. Nursing care. Checklist. Nursing process. Patient safety.

RESUMEN: Objetivos: Construir y validar los contenidos de un instrumento para registrar la sistematización de la atención de enfermería perioperatoria. Método: Estudio metodológico, realizado en un hospital universitario en el sur de Brasil, que incluyó revisión de literatura, mapeo cruzado entre observación no estructurada y taxonomías de la Asociación Norteamericana de Diagnóstico de Enfermería para la construcción de instrumentos y la aplicación de la técnica Delphi para validación, realizada entre noviembre y diciembre de 2018, con la disponibilidad de un formulario electrónico para diez expertos para

'Enfermeira pela Universidade Federal de Santa Catarina (UFSC). Residente de Enfermagem Multiprofissional em Saúde com Ênfase em Alta Complexidade no Hospital Universitário da UFSC - Florianópolis (SC), Brasil. ${ }^{2}$ Enfermeira; doutora em Enfermagem. Professora do Departamento de Enfermagem e do Programa de Pós-Graduação em Gestão do Cuidado em Enfermagem da UFSC - Florianópolis (SC), Brasil. ${ }^{3}$ Enfermeira, doutora em Enfermagem. Professora do Departamento de Enfermagem do Programa de Pós-Graduação da UFSC - Florianópolis (SC), Brasil.

${ }^{4}$ Enfermeira, mestre em Enfermagem. Enfermeira no Hospital Universitário da UFSC - Florianópolis (SC), Brasil.

*Autora correspondente: francielle.luciano@gmail.com

Recebido: 18/02/2019 - Aprovado: 26/09/2019

DOI: 10.5327/Z1414-4425201900040005 
evaluar la objetividad, claridad/comprensión, apariencia y viabilidad del contenido del instrumento, registrado en la escala Likert. Las respuestas obtenidas se enviaron al índice de validez de contenido (IVC), y las puntuaciones $\geq 0,8$ confirmaron la validación de contenido. Resultados: Los nueve grupos de información del instrumento fueron evaluados por enfermeras expertas. El IVC promedio obtenido entre todos los contenidos fue de 0.92 en la primera ronda de validación. Los resultados mostraron que la estrategia metodológica permitió la construcción de contenidos que representan la necesidad clínica de registros de enfermería perioperatoria. Conclusión: la implementación de un instrumento validado contribuye a una práctica de enfermería más segura y más calificada. Palabras clave: Centros quirúrgicos. Atención de enfermería. Lista de verificación. Proceso de enfermería. Seguridad del paciente.

\section{INTRODUÇÃO}

A sistematização da assistência em enfermagem (SAE) tem como objetivo identificar as situações de saúde-doença e as necessidades de cuidados de enfermagem, bem como auxiliar no desenvolvimento de intervenções, promoção, prevenção, recuperação e reabilitação da saúde do indivíduo, da família e da comunidade ${ }^{1}$.

Em 1990, no Brasil, foi proposta ${ }^{2}$ a aplicação do processo de enfermagem (PE) no cuidado de pacientes cirúrgicos nos períodos pré, trans e pós-operatório imediato (POI). O período pré-operatório divide-se em mediato e imediato, sendo o pré-operatório mediato desde o momento que se decide pela cirurgia até um dia antes do procedimento ${ }^{2}$. O pré-operatório imediato acontece nas 24 horas anteriores ao ato anestésico-cirúrgico, momento de preparação física e emocional do paciente e de sua família². O transoperatório compreende desde a entrada do paciente no centro cirúrgico (CC) até sua saída da sala de cirurgia, após o término do procedimento anestésico-cirúrgico ${ }^{2}$. O período pós-operatório imediato abrange as primeiras 24 horas após a cirurgia e inclui o tempo em que o paciente permanece na sala de recuperação pós-anestésica (SRPA) ${ }^{2}$.

Com um direcionamento ao cuidado ao paciente no período perioperatório, a sistematização da assistência de enfermagem perioperatória (SAEP) objetiva diminuir os riscos do ambiente do CC e da SRPA e promover quantidade e qualidade de materiais, equipamentos e recursos humanos. Esse processo compreende cinco etapas: avaliação pré-operatória de enfermagem, planejamento da assistência pré-operatória, implementação da assistência, avaliação da assistência por meio da visita pós-operatória de enfermagem e reformulação da assistência de acordo com os resultados obtidos ${ }^{3}$. Considera-se que o uso dos diagnósticos e das intervenções de enfermagem na SAEP é fundamental à prática, pois qualifica os cuidados e facilita a assistência de enfermagem prestada no POI de forma dinâmica, organizada e sistemática, o que exige do enfermeiro avaliação crítica e tomada de decisão ${ }^{4,5}$.
Orientando a SAEP e com o intuito de prevenir os eventos adversos, a Organização Mundial da Saúde (OMS) vem, desde 2004, mobilizando ações para a segurança do paciente durante os processos assistenciais. Destaca-se o desafio global Cirurgias Seguras Salvam Vidas, lançado pela OMS em 2008. A campanha consiste em uma proposta de checagem para cirurgia segura, realizada por meio de um instrumento objetivo, do tipo checklist, desenvolvido mediante revisões das práticas baseadas em evidências que identificaram as causas mais comuns de danos a pacientes no período perioperatório ${ }^{6,7}$.

Mesmo reconhecendo a efetividade do checklist de segurança na sala de cirurgia (adotado pelo cenário do estudo aqui apresentado), entende-se que também é de suma importância a atenta observação do paciente nas fases pré e pós-operatória, para o sucesso do procedimento e para a segurança do paciente. Sendo assim, faz-se necessário que os pacientes cirúrgicos recebam os cuidados durante todo esse processo, para o restabelecimento do equilíbrio fisiológico, o alívio da dor e dos desconfortos causados pela cirurgia, além da prevenção e da detecção de possíveis complicações ${ }^{6,7}$.

Em observações realizadas em um hospital de ensino do sul do Brasil, em 2017, identificou-se a necessidade da criação de um novo modelo de registro da SAEP, visto que o modelo utilizado estava desatualizado, incompleto e fragmentado e prolongava o tempo necessário para o preenchimento, fato que causou sentimento de inutilidade para os demais setores do hospital.

Entende-se que um instrumento para a segurança em cirurgia utilizado sistematicamente na unidade cirúrgica, na fase pré-operatória, no CC e na indução anestésica, durante a cirurgia, na SRPA e na unidade cirúrgica nas primeiras 24 horas do pós-operatório poderá contribuir para a maior segurança dos pacientes submetidos ao processo cirúrgico. Ainda, valorizará o trabalho da equipe de enfermagem de forma organizada e sequencial. Cabe, também, destacar que o cenário do estudo não adotava em suas práticas e registros a etapa dos diagnósticos de enfermagem no processo de enfermagem do CC. 
Considerando a obrigatoriedade da aplicação da $\mathrm{SAE}^{8}$ e seu desenvolvimento de maneira fragmentada na prática profissional do cenário do estudo, surgiu a motivação para a realização do presente estudo.

\section{OBJETIVOS}

Construir e validar conteúdos de um instrumento para registro da SAEP, em um hospital-escola do sul do Brasil.

\section{MÉTODO}

Trata-se de um estudo metodológico realizado em um hospital-escola situado na Região Sul do país que incluiu revisão da literatura, mapeamento cruzado entre a observação não estruturada e as taxonomias da North American Nursing Diagnosis Association (NANDA), para a construção dos conteúdos do instrumento e posterior aplicação da técnica Delphi para a validação de tais conteúdos. Inicialmente, realizou-se a observação não estruturada. Para tanto, a autora principal deste estudo, residente de enfermagem de alta complexidade no cenário do estudo, observou e registrou as necessidades de enfermagem verificadas no cotidiano da prática clínica, dificuldades de registro, queixas da equipe de enfermagem relacionadas aos instrumentos já utilizados, problemas e/ou necessidades em saúde e procedimentos cirúrgicos mais frequentes. Essa observação foi realizada no segundo semestre de 2017 e registrada em diário de campo. Sequencialmente, os achados foram agrupados, quando, então, foram identificados temas relevantes para a construção do instrumento.

Após essa etapa, realizou-se uma revisão da literatura, por meio da qual foram selecionadas publicações acerca dos temas: CC, cuidados de enfermagem, SAE, processo de enfermagem, segurança do paciente, paciente cirúrgico, SRPA e diagnóstico de enfermagem. Usaram-se as seguintes bases de dados e bibliotecas virtuais: Coordenação de Aperfeiçoamento de Pessoal de Nível Superior (Capes), Scientific Electronic Library Online (SciELO) e Biblioteca Virtual em Saúde (BVS).

A revisão de literatura também incluiu as taxonomias da NANDA International para a definição dos diagnósticos, resultados e intervenções de enfermagem ${ }^{8,9}$ que deveriam compor o instrumento. Essas taxonomias foram definidas por serem as adotadas no cenário do estudo.

Para a seleção dos diagnósticos de enfermagem, os problemas/as necessidades em saúde identificados e agrupados na observação não estruturada foram submetidos ao mapeamento cruzado $^{10}$ entre esses dados e os títulos dos diagnósticos da taxonomia NANDA-I ${ }^{8}$. Durante o cruzamento, registraram-se os títulos dos diagnósticos disponibilizados pela NANDA que se relacionavam com as necessidades da prática de enfermagem. Com base nesses diagnósticos, elegeram-se as intervenções recomendadas pela taxonomia NANDA/ Nursing Interventions Classification (NIC) $)^{9}$ para compor os conteúdos do instrumento.

Os dados da busca foram agrupados e registrados em quadros elaborados com auxílio do software Microsoft ${ }^{\circledR}$ Word.

Os resultados/conteúdos obtidos nas primeiras etapas metodológicas, somados aos instrumentos de registro da SAEP adotados no cenário do estudo (três instrumentos), foram organizados e adaptados junto a um processo criativo e experiência clínica das pesquisadoras do estudo, que elaboraram os conteúdos do instrumento que se apresenta neste artigo. A etapa de construção do instrumento ocorreu no primeiro semestre de 2018.

Para a validação dos conteúdos do instrumento, aplicou-se a técnica Delphi, uma ferramenta que proporciona o julgamento de informação de forma sistematizada, na busca do consenso de expertos (juízes avaliadores ou especialistas) em determinado tema para validação ${ }^{11}$. A técnica tem como objetivo a investigação de métodos para a coleta e organização dos dados, tais como: desenvolvimento, validação e avaliação de ferramentas e métodos de pesquisa, o que favorece a condução de investigações com grande rigor ${ }^{12}$. Geralmente é desenvolvida em rodadas de validação, e define-se o número de rodadas de acordo com o alcance dos índices de validação estabelecidos para o estudo ${ }^{13}$.

Estudos Delphi permitem identificar a falta e/ou a presença desnecessária de itens para melhor mensuração do objetivo, e esses aspectos somente podem ser perceptíveis por meio da validação de conteúdo por expertos na área em questão. Para tanto, é necessário elaborar e aplicar um questionário objetivo, estruturado ou não, no qual são apresentadas questões pertinentes, buscando o feedback do especialista, em rodadas subsequentes de avaliação. Em sua proposta original, o Delphi é, portanto, uma técnica para a busca de consenso entre opiniões de um grupo de especialistas sobre determinado evento/fenômeno ${ }^{13,14}$.

O estudo atendeu aos princípios éticos vigentes no Brasil e foi aprovado por Comitê de Ética em Pesquisa, de acordo com o Parecer $n^{\circ}$ 2.985.962, Certificado de Apresentação para Apreciação Ética (CAAE) n ${ }^{\circ} 92148218.3 .0000 .0121$, via Plataforma Brasil.

Os conteúdos em validação foram associados ao método de escalonamento de Likert ${ }^{14}$. Para a confirmação da validação 
dos conteúdos pelos juízes, foram calculados o percentual de concordância total e parcial e o índice de validade de conteúdo (IVC), considerando-se um IVC $\geq 0,80$ como valor mínimo para a validação dos conteúdos (média obtida entre todos os expertos). No caso de resultados abaixo desse valor, os conteúdos seriam revisados ou eliminados, conforme sugestão dos expertos.

O cálculo do IVC consiste na divisão do número total de especialistas que atribuíram os escores 3 (concordo parcialmente) e 4 (concordo) pelo número total de especialistas que participaram da rodada de validação.

Os participantes deste estudo foram enfermeiros expertos atuantes na área de enfermagem cirúrgica e vinculados ao cenário do estudo, tendo como critério de inclusão: experiência mínima de dois anos em clínica e/ou docência em enfermagem em clínica cirúrgica, CC e unidade de terapia intensiva; titulação mínima de mestrado; e atuação clínica no cenário do estudo. Realizou-se contato prévio presencial para esclarecimento do objetivo e do método de desenvolvimento do estudo e, ainda, para saber do interesse dos enfermeiros expertos em participar do estudo. Após a manifestação do interesse, foi aplicado o Termo de Consentimento Livre e Esclarecido e solicitada a assinatura dos participantes, confirmando sua inclusão no estudo.

O critério de exclusão estabelecido foi: enfermeiros que não deram retorno ao formulário online, ressaltando-se que os retornos parciais dos expertos não seriam excluídos. Ou seja, caso na primeira fase o experto desse retorno e na segunda etapa não o fizesse, cada avaliação seria considerada como resultado deste estudo para a análise estatística.

Nos estudos de validação, um dos pontos controversos refere-se ao número e à qualificação dos juízes, sendo recomendados o mínimo de cinco e o máximo de dez ${ }^{15}$. Neste estudo, estimou-se a inclusão mínima de dez juízes avaliadores em todas as rodadas de validação. Caso esse número não fosse atingido, novos expertos seriam convidados para inclusão no estudo.

Para a coleta de dados, elaborou-se um formulário eletrônico no serviço de armazenamento do Google Drive ${ }^{\circledR}$, contendo os tópicos que compõem o instrumento desenvolvido. Para cada conteúdo apresentado, foi inserida a escala Likert de quatro pontos (1 para discordo, 2 para discordo parcialmente, 3 para concordo parcialmente e 4 para concordo). O formulário também apresenta um espaço para registro de sugestões e/ou comentários dos avaliadores.

A validação dos itens abrangeu questões relacionadas aos conteúdos (apropriados, relevantes, exequíveis, conteúdo semântico), aparência (diagramação, distribuição dos elementos gráficos), clareza/ compreensão (conteúdo intuitivo, de fácil entendimento) e objetividade (conteúdos imparciais, diretos, práticos, claros).

O formulário foi enviado aos expertos após contato prévio por e-mail, complementando os esclarecimentos para o procedimento de validação do conteúdo, acordando o prazo máximo de entrega (15 dias) e disponibilizando o link de acesso ao instrumento de avaliação. As rodadas de validação ocorreram em novembro e dezembro de 2018.

Os resultados foram submetidos ao cálculo do IVC e apresentados na forma descritiva em tabela e quadro. A discussão dos dados foi sustentada por literatura científica atualizada e vinculada à temática.

\section{RESULTADOS}

Doze enfermeiros foram convidados para inclusão no estudo, e dez aceitaram. Destes, cinco atuavam como docentes no Departamento de Enfermagem de uma universidade no sul do Brasil, três no CC e dois na unidade de internação cirúrgica do cenário do estudo.

A idade dos expertos oscilou entre 27 e 58 anos (média de 41 anos); o tempo de formação variou entre seis e 37 anos (média de 17,5 anos); cinco eram mestres (50\%) e cinco doutores (50\%); e o tempo de experiência dos expertos no contexto perioperatório oscilou de dois a 20 anos (média de 11 anos).

Os resultados obtidos na observação não estruturada, na revisão de literatura, no mapeamento cruzado e na seleção e adaptação dos conteúdos para a composição do instrumento permitiram a elaboração dos conteúdos e da aparência do instrumento, intitulado "Processo de enfermagem: paciente cirúrgico".

Os conteúdos do instrumento foram agrupados nos seguintes tópicos $(\mathrm{T})$ :

- T1: Pré-operatório dados gerais;

- T2: Pré-operatório unidade de internação;

- T3: Pré-operatório CC;

- T4: Intraoperatório;

- T5: Pós-operatório imediato, SRPA;

- T6: Relatório de alta, SRPA;

- T7: Diagnóstico de enfermagem, POI, SRPA, enfermaria;

- T8: intervenções de enfermagem no pós-operatório imediato;

- T9: POI, enfermaria.

Quanto aos diagnósticos de enfermagem, elegeram-se 18 da taxonomia NANDA International ${ }^{8}$, inter-relacionados com 22 intervenções de enfermagem retiradas e adaptadas da 
NANDA/ NIC ${ }^{9}$. Ainda, reservou-se um espaço no instrumento para novas inclusões, conforme avaliação do enfermeiro.

Quanto à aparência do instrumento, optou-se pelas fontes Calibri Light e Calibri, tamanho 12 para os títulos e 11 para as demais informações, pela separação dos tópicos em quadros a partir dos títulos de cada tópico e pelo uso de figura. Os conteúdos foram distribuídos em duas folhas (quatro páginas - frente e verso) no formato de livreto. Esse formato foi pensado para que as folhas do instrumento não se separassem, considerando-se que o cenário do estudo ainda trabalha com prontuário impresso.

Na primeira rodada de validação, todos os tópicos avaliados atingiram o percentual de IVC $\geq 0,80$ de concordância, oscilando de 0,80 a 1,00 . O item que obteve o IVC mais próximo ao limite de 0,80 foi o de conteúdo. O item com IVC maior de concordância foi o de aparência, obtendo oito vezes a avaliação 1,00. O IVC final do instrumento, levando em conta todas as avaliações, alcançou a média geral de 0,92. Os percentuais de concordâncias parcial e total dos T1-T9 nos quesitos conteúdo, aparência, clareza/compreensão e objetividade e a totalidade dos IVC são apresentados na Tabela 1.

Mediante esses resultados, todos os conteúdos foram validados na primeira rodada de validação, entretanto algumas recomendações dos expertos foram consideradas relevantes e, portanto, agrupadas, analisadas e inseridas no instrumento. As recomendações dos expertos são apresentadas no Quadro 1.

Todos os conteúdos e a aparência do instrumento (duas folhas com conteúdos na frente e no verso) podem ser observados na íntegra no Apêndice 1.

\section{DISCUSSÃO}

Os resultados obtidos na validação do instrumento, objeto deste estudo, mostram que a estratégia metodológica proposta permitiu a construção de conteúdos que representam as necessidades clínicas para os registros da SAEP. Por esse motivo, o IVC mínimo necessário para validação foi alcançado na primeira rodada de avaliação, o que denota que os conteúdos representam a necessidade da prática clínica na percepção dos expertos e que a construção atendeu aos rigores científicos para produção de conhecimento e para a práxis.

Assim, evidencia-se que associar teoria, prática e articulação entre os profissionais fortalece a práxis, facilita o uso dos conhecimentos científicos, da ciência do cuidado clínico, contribuindo para incrementos na relação dialógica ${ }^{16}$ entre enfermeiros/enfermagem, pacientes e equipe de saúde, transformando e aprimorando o cuidado de enfermagem e seu registro.

As contribuições dos expertos foram, também, resultantes de sua experiência clínica no cenário do estudo, no contexto cirúrgico e com conhecimento científico relacionado, fatores essenciais para a conclusão deste estudo de validação. As melhorias nos conteúdos advindas das recomendações dos expertos se deram, na sua maioria, em relação à gramática $\mathrm{e}$ a disposição dos quadros, aprimorando o leiaute e tornando o instrumento mais agradável visualmente e de melhor preenchimento, o que facilitará seu uso por parte dos enfermeiros. O estudo afirma que essas alterações são pertinentes em estudos de validação, pois, mesmo tratando-se de avaliações subjetivas, tais alterações permitem melhor apresentação, compreensão dos conteúdos, clareza e objetividade do

Tabela 1. Índice de validade de conteúdo (IVC) do instrumento para registro da sistematização da assistência de enfermagem perioperatória. Florianópolis (SC), $2018(\mathrm{n}=10)$.

\begin{tabular}{|c|c|c|c|c|c|}
\hline \multirow{2}{*}{$\begin{array}{l}\text { Tópicos } \\
\text { avaliados }\end{array}$} & \multicolumn{4}{|c|}{ \% - Concordância Parcial - IVC \% - Concordância Total - IVC } & \multirow{2}{*}{ IVC } \\
\hline & Conteúdo & Aparência & Clareza/Compreensão & Objetividade & \\
\hline $\mathrm{T} 1$ & $40 \%-40 \%-0,8$ & $50 \%-50 \%-1,0$ & $40 \%-60 \%-1,0$ & $30 \%-70 \%-1,0$ & 0,92 \\
\hline $\mathrm{T} 2$ & $40 \%-40 \%-0,8$ & $20 \%-80 \%-1,0$ & $20 \%-70 \%-0,9$ & $20 \%-80 \%-1,0$ & 0,92 \\
\hline T3 & $0 \%-80 \%-0,8$ & $30 \%-60 \%-0,9$ & $20 \%-80 \%-1,0$ & $20 \%-80 \%-1,0$ & 0,92 \\
\hline T4 & $20 \%-60 \%-0,8$ & $0 \%-100 \%-1,0$ & $0 \%-80 \%-0,8$ & $20 \%-70 \%-0,9$ & 0,87 \\
\hline T5 & $20 \%-60 \%-0,8$ & $20 \%-80 \%-1,0$ & $20 \%-70 \%-0,9$ & $10 \%-80 \%-0,9$ & 0,90 \\
\hline T6 & $40 \%-50 \%-0,9$ & $10 \%-90 \%-1,0$ & $10 \%-80 \%-0,9$ & $10 \%-80 \%-0,9$ & 0,92 \\
\hline T7 & $30 \%-60 \%-0,9$ & $0 \%-100 \%-1,0$ & $0 \%-100 \%-1,0$ & $0 \%-100 \%-1,0$ & 0,97 \\
\hline T8 & $20 \%-70 \%-0,9$ & $10 \%-90 \%-1,0$ & $20 \%-80 \%-1,0$ & $10 \%-80 \%-0,9$ & 0,95 \\
\hline T9 & $40 \%-40 \%-0,8$ & $10 \%-90 \%-1,0$ & $30 \%-60 \%-0,9$ & $30 \%-70 \%-1,0$ & 0,92 \\
\hline Média & 0,83 & 0,98 & 0,93 & 0,95 & 0,92 \\
\hline
\end{tabular}

T1: pré-operatório dados gerais; T2: pré-operatório unidade de internação; T3: pré-operatório centro cirúrgico (CC); T4: intraoperatório; T5: pós-operatório imediato, sala de recuperação pósanestésica (SRPA); T6: relatório de alta, SRPA; T7: diagnóstico de enfermagem, períodos pré, trans e pós-operatório imediato (POI), SRPA e enfermaria; T8: intervenções de enfermagem no POI; T9: POI, enfermaria. 
instrumento, bem como facilitam a leitura, a interpretação dos conteúdos e a objetividade do instrumento ${ }^{17}$.

Em relação aos diagnósticos e às intervenções de enfermagem, as recomendações dos expertos foram consideradas pertinentes para o cuidado dos pacientes cirúrgicos. Com a inclusão, totalizaram-se 22 diagnósticos e 24 intervenções de enfermagem. Os diagnósticos incluídos são baseados em problemas reais e/ou potenciais, facilitando a linha de pensamento para o desenvolvimento das intervenções vinculadas a eles, a fim de que os resultados de enfermagem configurem as melhores respostas. A utilização dos diagnósticos e das intervenções propostas pela NANDA otimiza o tempo

Quadro 1. Recomendações dos expertos para alterações no instrumento. Florianópolis (SC), 2018.

\begin{tabular}{|c|c|}
\hline $\begin{array}{l}\text { Tópicos } \\
\text { avaliados }\end{array}$ & Recomendações dos expertos \\
\hline T1 & $\begin{array}{l}\text { - Adicionar "Falta de equipe médica" nos motivos } \\
\text { de suspensão de cirurgias; } \\
\text { - Incluir item "Infecções" nas comorbidades; } \\
\text { - Adicionar "( ) Sim ( ) Não" no item "Cirurgia } \\
\text { suspensa"; } \\
\text { - Alterar "Não tem vaga de UTI" por "Falta de leito } \\
\text { de UTI". }\end{array}$ \\
\hline T2 & $\begin{array}{l}\text { - Adicionar item "Reserva de sangue"; } \\
\text { - Adicionar item “Realizada higiene oral”. }\end{array}$ \\
\hline T3 & $\begin{array}{l}\text { - Adicionar linha para escrita de "Outros dispositi- } \\
\text { vos invasivos". }\end{array}$ \\
\hline T4 & $\begin{array}{l}\text { - Trocar "Sistema de aquecimento disponível" por } \\
\text { "Sistema de aquecimento instalado"; } \\
\text { - Alterar "Balanço" por "Volume total"; } \\
\text { - No item "Anestesia", adicionar uma linha para ano- } \\
\text { tações sobre a anestesia e a palavra "Entubação"; } \\
\text { - Acrescentar na tabela de conferências o item “N/A". }\end{array}$ \\
\hline T5 & - Nenhuma alteração. \\
\hline T6 & • Trocar palavra “Secreções” por “Drenagem”. \\
\hline $\mathrm{T7}$ & $\begin{array}{l}\text { - Adicionar “Diagnóstico de enfermagem”, “Risco } \\
\text { de lesão por pressão", “Risco de confusão aguda”, } \\
\text { “Risco de infecção no sítio cirúrgico" e “Risco de } \\
\text { hipotermia perioperatória”; } \\
\text { - Adicionar linhas extras, caso haja necessidade de } \\
\text { outros diagnósticos. }\end{array}$ \\
\hline T8 & $\begin{array}{l}\text { - Adicionar a palavra "Registrar" no cuidado sobre } \\
\text { sangramento; } \\
\text { - Adicionar "Realizar cuidados com fluidoterapia" e } \\
\text { "Atentar para sinais de sintomas de hipotermia". }\end{array}$ \\
\hline T9 & - Nenhuma alteração. \\
\hline
\end{tabular}

T1: pré-operatório dados gerais; T2: pré-operatório unidade de internação; T3: pré-operatório centro cirúrgico (CC); T4: intraoperatório; T5: pós-operatório imediato, sala de recuperação pós-anestésica (SRPA); T6: relatório de alta, SRPA; T7: diagnóstico de enfermagem, períodos pré, trans e pós-operatório imediato (POI), SRPA e enfermaria; T8: intervenções de enfermagem no POI; T9: POI, enfermaria; UTI: unidade de terapia intensiva; N/A: nenhuma das alternativas. de elaboração dos diagnósticos de enfermagem e colabora na decisão dos melhores cuidados a serem prestados, além de padronizar a prática e reduzir o tempo despendido pelos enfermeiros com os diagnósticos de enfermagem ${ }^{18}$.

Cabe destacar que o cenário do estudo não atuava com uma taxonomia para o uso dos diagnósticos de enfermagem no CC. Com a implantação desse novo instrumento, haverá a padronização da linguagem e da comunicação entre enfermeiro e equipe de enfermagem.

Aponta-se que uma das dificuldades encontradas na construção do instrumento foi o agrupamento do conteúdo necessário e dos itens mais relevantes no menor espaço possível, para a execução da SAEP e o adequado registro das ações de enfermagem. Dividido em nove partes, considera-se que o instrumento é de fácil utilização e inclui conteúdos abrangentes e significativos à prática clínica no contexto cirúrgico, uma vez que cada profissional é responsável pelo preenchimento das informações pertinentes ao seu respectivo setor de atendimento ao paciente e pode preenchê-las de forma rápida, já que o instrumento é disposto de forma simples e objetiva.

A utilização de instrumentos assistenciais pelo enfermeiro deve estar aliada ao conhecimento técnico e científico sistematizado sobre as ações a serem realizadas durante o período perioperatório. Além disso, as definições de ações a serem executadas contribuem para que haja maior sincronismo e eficácia entre os diversos profissionais e, portanto, é esperada maior probabilidade de sucesso nas intervenções ${ }^{19}$.

Registram-se, como limitações deste estudo, a não inclusão de expertos externos ao cenário do estudo, a não aplicação de procedimentos analíticos e a não inclusão de todos os conteúdos do checklist de cirurgia segura. Esse último aspecto justifica-se por ter sido uma decisão dos profissionais do cenário do estudo, pois, nesse contexto, um instrumento próprio para esse fim é aplicado.

Considera-se que o instrumento produzido e validado neste estudo contribuirá para uma assistência de enfermagem mais segura, bem como para a valorização do trabalho prestado pelos enfermeiros no período perioperatório.

Por fim, entende-se que o presente estudo é relevante por construir e validar um instrumento, propondo uma assistência mais segura para pacientes cirúrgicos e, ainda, promovendo maior visibilidade do trabalho do enfermeiro e da equipe de enfermagem nesse processo. Além disso, permite um continuum no cenário do estudo ao sugerir um instrumento único a todas as etapas da SAEP, organizando e padronizando os registros de enfermagem.

Outro trabalho semelhante a esta investigação aponta para a importância de estudos que associem a academia e as necessidades 
da clínica, ou seja, teoria e prática. Ainda, ressalta que a relevância de investigações desse porte está no fato de elas atenderem às reais necessidades do serviço, possibilitando a valorização da profissão de enfermagem e a segurança do paciente 4 .

\section{CONCLUSÃO}

A versão final do instrumento foi validada por 10 expertos para a implementação do registro das fases da SAEP no cenário do estudo - um hospital-escola no sul do Brasil —, e a média do IVC obtido entre todos os conteúdos foi de 0,92 já na primeira rodada de validação. Ressalta-se que as contribuições dos expertos foram essenciais, uma vez que proporcionaram a construção de um instrumento adequado ao cotidiano do enfermeiro cirúrgico, com a utilização de termos mais coerentes, atualizados e de fácil preenchimento, facilitando sua adesão.

Recomenda-se validação de conteúdo com aplicação de procedimentos analíticos e com expertos externos ao cenário do estudo.

\section{REFERÊNCIAS}

1. Silva JP, Garanhani ML, Peres AM. Systematization of nursing care in undergraduate training: the perspective of complex thinking. Rev Latino-Am Enfermagem. 2015;23(1):59-66. http://dx.doi. org/10.1590/0104-1169.0096.2525

2. Associação Brasileira de Enfermeiros de Centro Cirúrgico, Recuperação Anestésica e Centro de Material e Esterilização. Diretrizes de práticas em enfermagem cirúrgica e processamento de produtos para a saúde. 7ª ed. São Paulo: SOBECC; 2017.

3. Saraiva EL, Sousa CS. Critically IIl patients in the postanesthesia care unit: integrative review. Rev SOBECC. 2015;20(2):104-12. http:// dx.doi.org/10.5327/Z1414-4425201500020006

4. Monteiro EM, Melo CL, Amaral TLM, Prado PR. Cirurgias seguras: elaboração de um instrumento de enfermagem perioperatória. Rev SOBECC. 2014;19(2):99-109. http://dx.doi.org/10.4322/sobecc.2014.016

5. Ribeiro CP, Silveira CO, Benetti ER, Gomes JS, Stumm EM. Diagnósticos de enfermagem em pacientes no pós-operatório de cirurgia cardíaca. Rev Rene. 2015;16(2):159-67. http://dx.doi. org/10.15253/2175-6783.2015000200004

6. Organização Mundial da Saúde. Segundo desafio global para a segurança do paciente. Manual cirurgias seguras salvam vidas. Genebra: Organização Mundial da Saúde; 2009.

7. Alpendre F, Cruz E, Dyniewicz A, Mantovani M, Silva A, Santos G. Safe surgery: validation of pre and postoperative checklists. Rev Latino-Am Enfermagem. 2017;25:e2907. http://dx.doi. org/10.1590/1518-8345.1854.2907

8. Herdman TH, Kamitsuru S. North American Nursing Diagnosis Association. Diagnósticos de enfermagem da NANDA-I: definições e classificação: 2018-2020. $11^{\text {a }}$ ed. Porto Alegre: Artmed; 2018.

9. Bulechek GM, Butcher HK, Dochtermn J, Wagner CM. NIC - Classificação das Intervenções de Enfermagem. 6 ${ }^{\mathrm{a}}$ ed. Rio de Janeiro: Elsevier; 2016.

10. Morais SCRV, Nóbrega MML, Carvalho EC. Cross-mapping of results and Nursing Interventions: contribution to the practice. Rev Bras Enferm. 2018;71(4):1883-90. http://dx.doi.org/10.1590/0034-7167-2017-0324

11. Marques JBV, Freitas D. Método Delphi: caracterização e potencialidades na pesquisa em educação. Pro-Posições. 2018;29(2):389-415. http:// dx.doi.org/10.1590/1980-6248-2015-0140
12. Crozeta K, Roehrs H, Stocco JGD, Meier MJ. Pesquisa metodológica: novos e velhos desafios. In: Anais da 17. Semana Nacional de Pesquisa em Enfermagem [Internet]; 2013 [acessado em 20 dez. 2018]. Rio Grande do Norte: Associação Brasileira de Enfermagem; 2013. p. 1151-3. Disponivel em: http://www.abeneventos.com.br/ anais_senpe/17senpe/pdf/0835po.pdf

13. Rozados HBF. $\mathrm{O}$ uso da técnica Delphi como alternativa metodológica para a área da Ciência da Informação. Em Questão [Internet]. 2015 [acessado em 3 abr. 2019];21(3):64-86. Disponível em: https://seer. ufrgs.br/EmQuestao/article/viewFile/58422/36043

14. Pereira RDM, Alvim NAT. Delphi technique in dialogue with nurses on acupuncture as a proposed nursing intervention. Rev Esc Anna Nery. 2015;19(1):174-80. http://dx.doi.org/10.5935/ $1414-8145.20150024$

15. Rodrigues AB, Cunha GH, Aquino CBQ, Rocha SR, Mendes CRS, Firmeza MA, et al. Head and neck cancer: validation of a data collection instrument. Rev Bras Enferm. 2018;71(4):1899-906. http://dx.doi. org/10.1590/0034-7167-2017-0227

16. Nyholm L, Salmela S, Nyström L. Application in the world of understanding: researchers' experiences of participation in reflective dialogues. [Internet]. 2018 [acessado em 8 nov. 2018];5. Disponível em: http://dx.doi.org/10.1177/2333393618815006

17. Pompeo DA, Rossi LA, Paiva L. Content validation of the nursing diagnosis nausea. Rev Esc Enferm USP. 2014:48(1):48-56. http:// dx.doi.org/10.1590/S0080-623420140000100006

18. Bertoncello KCG, Sávio B, Ferreira JM, Amante LN, Nascimento ERP. Nursing diagnoses and proposals for nursing interventions for patients in the immediate post-operative period following elective surgery. Cogitare Enferm. 2014;19(3):534-45. http://dx.doi.org/10.5380/ ce.v19i3.33676

19. Lemos CS, Poveda VB, Peniche ACG. Construction and validation of a nursing care protocol in anesthesia. Rev Latino-Am Enfermagem. 2017;25:e2952. http://dx.doi.org/10.1590/ $1518-8345.2143 .2952$

20. Galdeano LE, Rossi LA, Nobre LF, Ignácio DS. Diagnósticos de enfermagem de pacientes no período transoperatório de cirurgia cardíaca. Rev Latino-Am Enfermagem. 2003;11(2):199-206. Disponível em: http://dx.doi.org/10.1590/S0104-11692003000200009 
Apêndice 1. Instrumento validado para o registro da sistematização da assistência de enfermagem perioperatória. Florianópolis-SC, 2018.

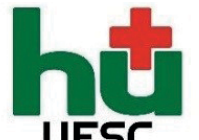

\section{HOSPITAL POLYDORO HERNANI DE SÃO THIAGO} UNIVERSIDADE FEDERAL DE SANTA CATARINA

\section{PROCESSO DE ENFERMAGEM} PACIENTE CIRÚRGICO

Nome: Quarto/Leito Registro:

DN: $/ 1$ Idade: Raça: Sexo: Altura:
Peso: Cond. UTI: $\square \operatorname{Sim} \square$ Não

Diagnóstico Médico: Cirurgia Proposta: Data Cirurgia: $\quad / 1$

\begin{tabular}{|lll}
\hline Comorbidades / Vícios / Informações importantes \\
\hline$\square$ HAS & $\square$ Gravidez & $\square$ D. pulmonar \\
$\square$ DM & $\square$ Sepse & $\square$ D. hematológica \\
$\square$ Tabagismo & $\square$ D. coronariana & $\square$ D. tireoide \\
$\square$ Etilismo & $\square$ Disritmia & $\square$ Alergias \\
$\square$ Substância llícita & $\square$ D. Renal & $\square$ Infecções \\
\hline
\end{tabular}

\begin{tabular}{|c|c|c|}
\hline Cirurgia: & \multicolumn{2}{|c|}{ Unidade de internação: } \\
\hline 口Eletiva & $\square \mathrm{CMI}$ & 口UTI \\
\hline DUrgência & 口CMII & $\square$ Gineco \\
\hline \multirow[t]{2}{*}{$\square$ Emergência } & $\square \mathrm{UICl}$ & $\square$ Emergência \\
\hline & DUICll & $\square$ Externo \\
\hline \multicolumn{2}{|c|}{ Cirurgia suspensa: } & $\square$ Não \\
\hline \multicolumn{3}{|c|}{$\square$ Falta de leito de UTI } \\
\hline \multicolumn{3}{|c|}{$\square$ Decisão da equipe médica } \\
\hline \multicolumn{3}{|c|}{$\square$ Falta de equipe: enfermagem/médica } \\
\hline \multicolumn{3}{|c|}{$\square$ Erro na estimativa do tempo de cirurgia } \\
\hline \multicolumn{3}{|c|}{$\square$ Falta de material } \\
\hline \multicolumn{3}{|c|}{$\square$ Paciente sem condições clínicas } \\
\hline
\end{tabular}
PRÉ-OPERATÓRIO - UNIDADE DE INTERNAÇÃO

$\square$ Jejum pré-operatório - desde:__ hs $\quad$ hPreparo especial:

$\square$ Exames de pré-operatórios disponíveis (impressos ou no sistema HU)

$\square$ Termo de consentimento preenchido e assinado: $\square$ Cirúrgico $\square$ Anestésico

$\square$ Realizado banho pré-cirúrgico com:

$\square$ Reserva de sangue

$\square$ Realizada higiene oral

$\square$ Realizada tricotomia às hs (Máx. 2 hs antes da cirurgia) $\square$ Unidade Internação $\square$ Centro cirúrgico

$\square$ Remoção de adereços/pertences pessoais $\quad \square$ Remoção de próteses

$\square$ Demarcação de sítio cirúrgico - Lateralidade: $\square$ Direito(a) $\square$ Esquerdo(a) $\square \mathrm{N} / \mathrm{A}$

$\square$ Orientado sobre cirurgia/cuidados perioperatórios

$\square$ Prontuário acompanha o paciente

\section{Avaliação de enfermagem:}

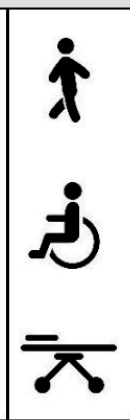

\begin{tabular}{llllllll} 
Avallação de enfermagem. \\
\hline \\
\hline Sinais Vitais: PA: & $\mathrm{mmHg}$ & $\mathrm{FC}:$ & $\mathrm{bpm}$ & $\mathrm{FR}:$ & $\mathrm{mrpm}$ & $\mathrm{T}:$ & \\
\hline
\end{tabular}

Enfermeiro responsável / COREN:

\section{PRÉ-OPERATÓRIO CENTRO CIRÚRGICO}

Paciente e equipe confirmam as informações:

Identidade (nome completo, data de nascimento e prontuário):

Confirmação/demarcação de sítio cirúrgico - Lateralidade (se houver):

$\square \operatorname{Sim} \square$ Não $\square$ N/A

Termo de Consentimento da cirurgia assinado

$\square$ Direito(a)

$\square$ Esquerdo(a)

$\square N / A$

Fluidoterapia: $\quad \square$ Acesso venoso adequado

$\square \operatorname{Sim} \square$ Não $\square$ N/A

Outros dispositivos invasivos:

Alergias: $\square$ Nega/desconhece $\square$ Sim - Qual(is) 
Apêndice 1. Continuação.

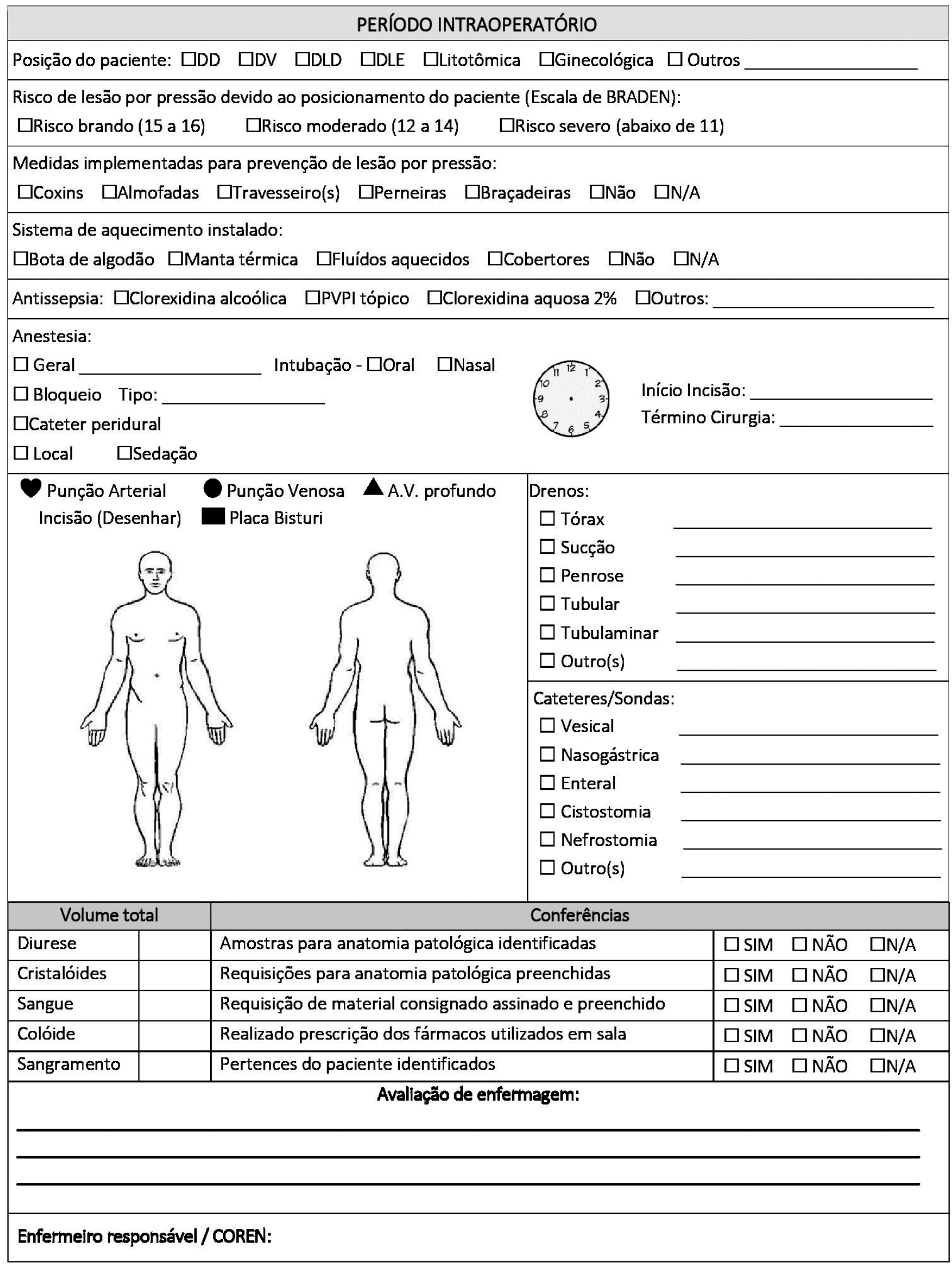


Apêndice 1.Continuação.

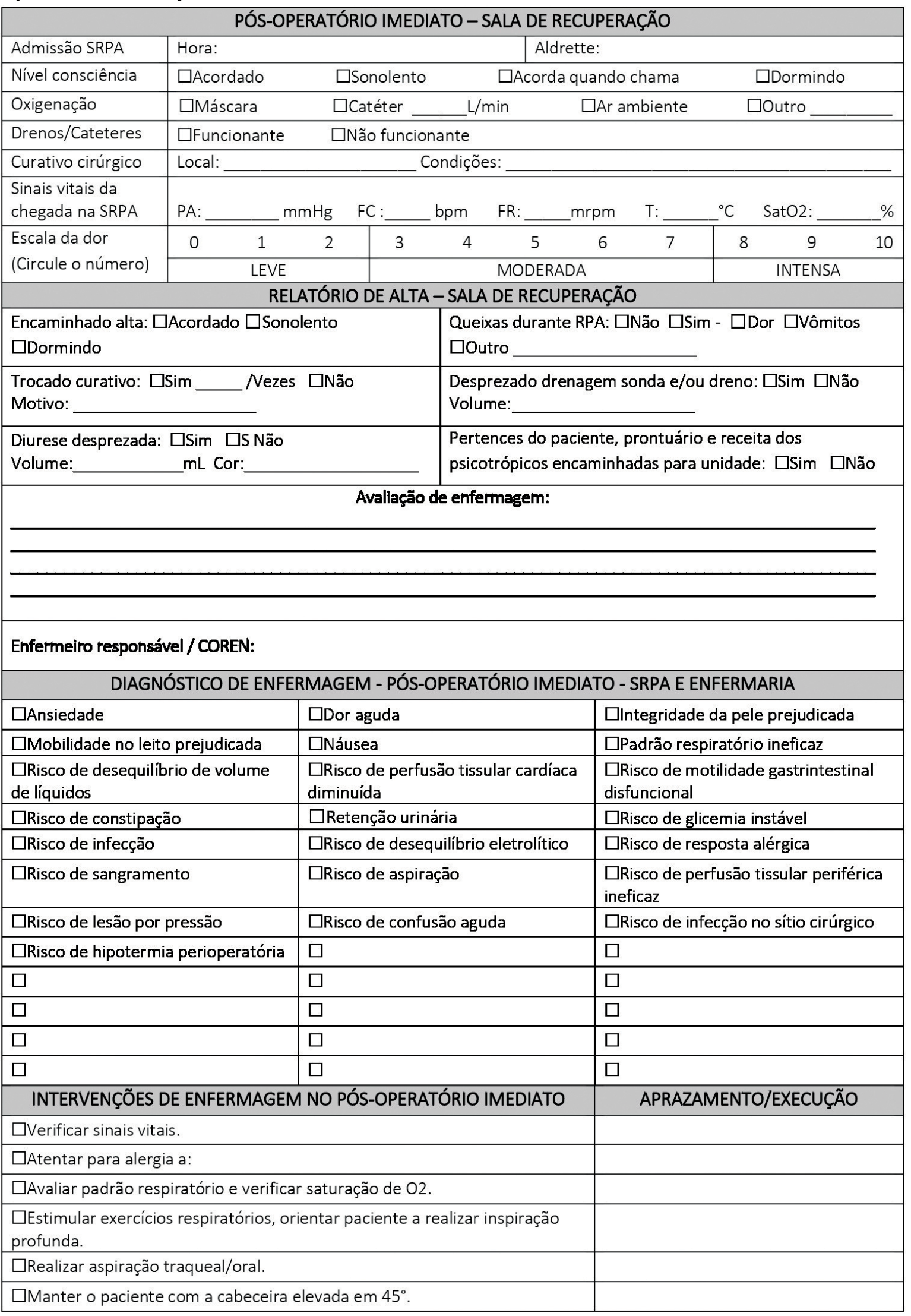


Apêndice 1. Continuação.

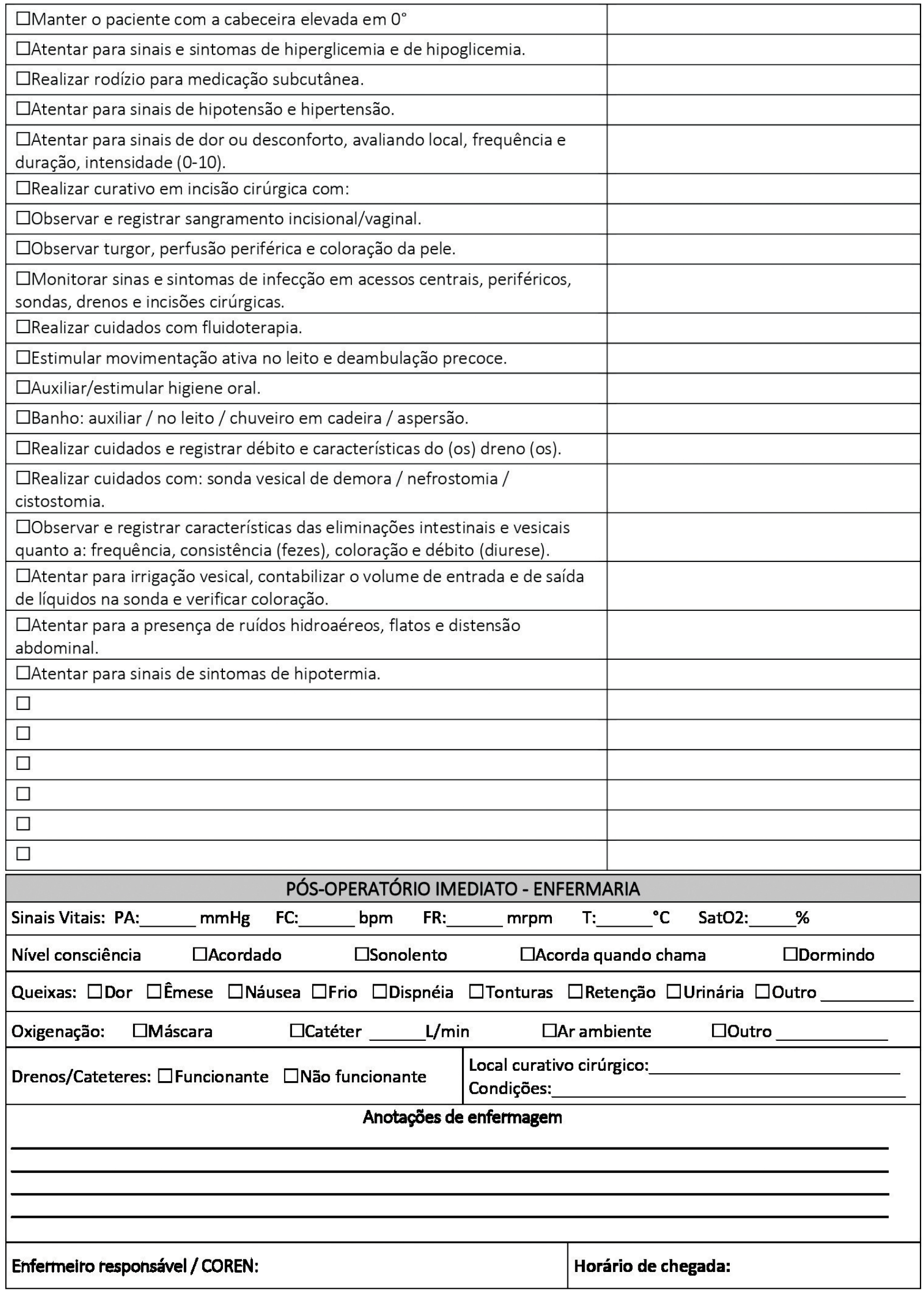

